\title{
Sensitivity of the Human Binaural Cortical Steady State Response to Interaural Level Differences
}

\author{
Sarah Massoud, ${ }^{1}$ Steve J. Aiken, ${ }^{1,2,3}$ Aaron J. Newman,,${ }^{1,2,4,5}$ Dennis P. Phillips, ${ }^{1,2,5}$ \\ and Manohar Bance B, $^{2,5}$
}

\begin{abstract}
Objectives: Periodic alternations of the interaural correlation of a noise stimulus evoke an auditory steady state response that can be measured at the scalp, providing an objective measure of binaural integration. The purpose of this study was to examine the effect of interaural level differences on this steady state response.
\end{abstract}

Design: Auditory steady state responses at 4 and $8 \mathrm{~Hz}$ were recorded to $4 \mathrm{~Hz}$ cycles of interaural correlation change of a Gaussian noise in normal-hearing listeners. Responses were recorded with symmetric presentation levels of 80,60 , and $40 \mathrm{~dB}$ SPL and with interaural asymmetries ranging from 10 to $40 \mathrm{~dB}$, varying in 10-dB steps.

Results: The $8 \mathrm{~Hz}$ response was sensitive to interaural level asymmetry and fell to $50 \%$ strength at an asymmetry of $18 \mathrm{~dB}$, although the response was detectable to an asymmetry of $30 \mathrm{~dB}$. A simultaneously present $4 \mathrm{~Hz}$ response showed no sensitivity to interaural level difference. Significant responses were recorded in all participants.

Conclusions: The $8 \mathrm{~Hz}$ auditory steady state response to a $4 \mathrm{~Hz}$ change in noise interaural correlation might be useful as an objective measure of binaural integration in asymmetric hearing loss. Response amplitude is more negatively affected by small amounts of interaural asymmetry than by large reductions in overall presentation level.

(Ear \& Hearing 2011;32;114-120)

\section{INTRODUCTION}

When human listeners are presented with a binaural noise stimulus and an interaural delay is introduced, there is a perceptible shift in stimulus laterality toward the ear receiving the time-leading stimulus and an evoked triphasic P1-N1-P2 response that can be recorded at the scalp (Halliday \& Callaway 1978; McEvoy et al. 1990; Picton et al. 1991). Although the evoked response has its origins in the cortex (Hari et al. 1980; Scherg et al. 1989), the response depends on input from subcortical low-frequency central neurons that are sensitive to interaural phase differences (Yin et al. 1986); these are part of a larger architecture for the coding of the cues for sound source azimuth (Phillips 2008).

Similar responses can be recorded when the interaural correlation of a noise changes abruptly (Chait et al. 2005; Dajani \& Picton 2006). The two types of change give rise to different perceptual experiences: changes in interaural timing affect the perceived location or lateralization of a sound, whereas changes in interaural correlation affect the perceived compactness or diffuseness of a sound (Blauert \& Lindemann 1986). However, interaural timing and interaural correlation change responses are both dependent on subcortical neurons with precise spike timing and phase-sensitive inputs from the two ears (e.g., the medial superior olive; Moore 1991).

Departments of ${ }^{1}$ Psychology and ${ }^{2}$ Surgery, ${ }^{3}$ School of Human Communication Disorders, ${ }^{4}$ Department of Psychiatry, and ${ }^{5}$ Neuroscience Institute, Dalhousie University, Halifax, Nova Scotia, Canada.
Dajani and Picton (2006) recently described a human auditory steady state response (ASSR) evoked by a wideband noise stimulus that periodically alternated in interaural correlation between a value of 0 and +1 . At slow presentation rates (two changes per second), a clear P1-N1-P2 complex occurred after each change in interaural correlation, but at faster rates, a steady state response emerged. With $4 \mathrm{~Hz}$ cycles of correlation change (i.e., eight correlation changes per second), the dominant frequency present in the ASSR was $8 \mathrm{~Hz}$. It is possible that the ASSR in this case reflected transient cortical P1-N1-P2 responses time-locked to each change in interaural correlation, because the match between the P1N1-P2 peak latencies and the stimulus period could result in each $\mathrm{P} 1$ response temporally overlaying the $\mathrm{P} 2$ response to the preceding change in correlation. This interpretation is compatible with magnetoencephalography data indicating that forebrain auditory responses are driven directly by both directions of interaural correlation change (Chait et al. 2005). Alternatively, the steady state response might be distinct from transient responses recorded at slower presentation rates, because the latter have significant refractory periods (Näätänen \& Picton 1987).

Several methods have been proposed for the objective assessment of binaural temporal integration using electrophysiologic analogs of the binaural masking level difference (i.e., the improvement in hearing thresholds that occurs when the interaural correlation of a signal is different from the interaural correlation of a simultaneous masker); these involve recording steady state responses to low-frequency tones from the brain stem (Wilson \& Krishnan 2005), or steady state responses to amplitude-modulated tones from the cortex (Wong \& Stapells 2004), and measuring differences in response amplitude as a function of the phase of the signal or masker. The interaural correlation ASSR is probably also related to the binaural masking level difference, given that the masking level difference is closely related to interaural correlation change detection (Durlach et al. 1986; Koehnke et al. 1986, 1995; Jain et al. 1991; Bernstein \& Trahiotis 1992; Culling et al. 2001; Krumbholz et al. 2009), but a key advantage of the interaural correlation ASSR approach is that the response - not the amplitude difference between two responses-provides evidence of binaural integration. Moreover, the interaural correlation of a broadband noise can be changed without introducing any monaurally detectable changes: when listening with only one ear, the noise is perceived as steady, and no electrophysiologic responses are recorded when the correlation changes (Dajani \& Picton 2006). Thus, the response unequivocally reflects binaural temporal integration. The steady state nature of the response also simplifies detection. Any significant 
activity at the frequency or harmonics of the correlation change provides direct evidence of binaural processing.

One potentially important application of this ASSR is its utility as a simple, objective measure of binaural temporal integration for individuals with asymmetric hearing loss. Binaural integration is critical for optimal hearing in noise and complex auditory environments (Gatehouse \& Noble 2004; Boothroyd 2006) but is easily compromised by hearing loss (Häusler et al. 1983; Bronkhorst \& Plomp 1989), particularly when hearing thresholds are asymmetric (Bocca \& Antonelli 1976; Olsen et al. 1976; Wilson et al. 1985; Bronkhorst \& Plomp 1989).

Understanding binaural temporal integration, particularly the levels of asymmetry at which it breaks down, has implications for the treatment of asymmetric loss (Nia \& Bance 2001). For instance, when deciding whether to surgically correct conductive hearing loss, many surgeons will not attempt to correct an ear that cannot be brought to within $15 \mathrm{~dB}$ of the contralateral ear's hearing level or to within $30 \mathrm{~dB}$ absolute hearing level. This is based on an understanding that hearing is an innately binaural process, and that the real-world functional impact of the hearing gain in the operated ear will depend on the hearing level in the other (nonoperated) ear. This " $15 / 30$ " surgical rule is sometimes called the Belfast Rule of Thumb (Smyth \& Patterson 1985; Toner \& Smyth 1993) and is also alluded to in other methods of reporting hearing benefit from middle ear surgery, such as the Glasgow Benefit Plot (Browning et al. 1991; Browning 1993). These rules are based on hearing handicap or questionnaire-type data, correlating patient perceived satisfaction with the hearing gain achieved, and there are broad variances in the data. What is lacking to date is the use of a physiologic objective parameter that definitely signals binaural integration and the level of asymmetry at which it breaks down. The ability to measure this would allow us to probe many of these rules of thumb from a physiologic perspective.

An objective measure would also be useful for studying longitudinal changes in binaural integration after surgical interventions that increase or decrease interaural symmetry. There is evidence that the nervous system can compensate for longstanding asymmetries by perceptually centering sounds presented at equal or nearly equal SPLs to each ear (Durlach et al. 1981; Wilson et al. 1985); optimal binaural temporal integration might similarly occur at equal or nearly equal SPLs (Olsen et al. 1976). One study found that individuals with asymmetric conductive loss had normal masking level differences after they had adjusted interaural stimulus levels to center the stimulus perceptually (Quaranta \& Cervellera 1974), but the authors did not report the final stimulus SPLs. If these had been equal, any attempt to compensate for the asymmetry might have interfered with binaural processing (Wilson et al. 1985) at least temporarily.

The effects of hearing asymmetries and corrective treatments on binaural temporal integration could be clarified by recording binaurally sensitive ASSRs in patients and in normal-hearing people at multiple levels of interaural symmetry (i.e., by varying the levels presented to each ear), with the amplitudes derived from the steady state response used as indices of binaural temporal integration at each level of symmetry. Furthermore, these measures could be made before treatment and at regular post-treatment intervals. As a first step in the development of such an application, this study measured ASSRs in response to $4 \mathrm{~Hz}$ cycles of interaural correlation change (i.e., eight changes per second) as a function of interaural stimulus level and symmetry in a group of normalhearing listeners. The objective of the study was to measure the sensitivity of the interaural correlation ASSR to interaural level changes at the correlation cycle frequency $(4 \mathrm{~Hz})$, as well as at the second harmonic of this frequency (i.e., the frequency of correlation change), at which Dajani and Picton (2006) obtained the largest responses.

\section{MATERIALS AND METHODS}

\section{Participants}

Fifteen young adult participants (12 females) were recruited from Dalhousie University through word of mouth. All participants had normal hearing, which is defined as tone thresholds of $15 \mathrm{~dB}$ HL or better at octave intervals from 250 to $8000 \mathrm{~Hz}$. All testing was carried out with the approval of the Capital District Health Authority Research Ethics Board. Informed consent was obtained from each participant before data collection.

\section{Stimuli}

Stimuli were created in MATLAB (The MathWorks, Inc., Natick, MA) following the general design described by Dajani and Picton (2006). Sixteen short samples of broadband Gaussian noise were created and concatenated to produce an ongoing binaural stimulus, with the interaural correlation $(\rho)$ alternating abruptly between 0 and +1 every 125 msecs. An interaural correlation of 0 was approximated by using independent noise samples for each ear, and an interaural correlation of 1 was achieved by using identical noise samples for each ear. When listening to either ear independently, only a steady noise could be heard. When listening to both ears simultaneously, the correlation change could be easily heard as an oscillation between a compact noise (at $\rho=+1$ ) and a diffuse noise (at $\rho=0$ ).

\section{Procedure}

ASSR recordings were blocked by level of asymmetry and absolute level. Each block was of 4 mins duration during which the correlation between stimuli at the two ears alternated at a rate of $8 \mathrm{~Hz}$, such that one complete cycle of IAC $=0 /+1$ occurred four times per second. For all blocks, the sound level was kept constant in one ear while the signal in the other ear was decreased (at a fixed level per block). Both 80 and $60 \mathrm{dBA}$ were used as reference points (calibrated in a $2-\mathrm{cm}^{3}$ coupler), i.e., the sound was kept constant in one ear either at 80 or at 60 $\mathrm{dBA}$; the sound intensity in the opposite ear was decreased in $10-\mathrm{dB}$ steps from 80 or $60 \mathrm{dBA}$ to $40 \mathrm{dBA}$. The final condition tested was a presentation at $40 \mathrm{dBA}$ in both ears. We anticipated that $40 \mathrm{dBA}$ would be the lowest stimulus level likely to evoke a detectable ASSR. Thus, five asymmetry levels were tested with a reference level of $80 \mathrm{dBA}(80: 80,80: 70,80: 60$, $80: 50$, and 80:40, resulting in asymmetries of $0,10,20,30$, and $40 \mathrm{~dB}$ ), whereas only three asymmetry levels were tested with a reference level of $60 \mathrm{dBA}(60: 60,60: 50$, and 60:40). The use of two reference levels enabled us to tease out whether it was the level of asymmetry or the absolute level of the stimuli that 
limited effective binaural temporal integration. Each condition was presented in two separate blocks. For all asymmetrical conditions, the ear with the reference (i.e., more intense) level was counterbalanced across blocks.

ASSR recordings were made in a quiet room. Participants relaxed in a reclining chair to eliminate artifacts arising from muscle tension in the neck and watched a movie with subtitles and no sound during the experiment. Stimuli were presented through Bio-logic ER-3A electrically shielded insert earphones (Natus Medical Inc., San Carlos, CA).

Responses were recorded between the vertex and the left mastoid, filtered between 0.1 and $100 \mathrm{~Hz}$, and amplified 10,000 times using a Grass LP-511 electrophysiological amplifier (Grass Technologies, West Warwick, RI). All interelectrode impedances were maintained below $5 \mathrm{k} \Omega$ and within $1 \mathrm{k} \Omega$ of each other. Custom software for data collection "Auditory Response Toolkit 1.0" was used in conjunction with LabVIEW and an M Series PCI model data acquisition card (National Instruments Corporation, Austin, TX) to present stimuli and record responses in a time-locked fashion. A sampling rate of $32 \mathrm{kHz}$ was used for stimulus presentation and for response recording. Data were averaged in 1-sec epochs and stored to disk along with the continuous (unaveraged) recording.

\section{Analysis}

The unaveraged data file from each block contained 250 epochs, each $1 \mathrm{sec}$ in length. These were Fourier transformed, and the noise power in each epoch was estimated by averaging the power in four $1-\mathrm{Hz}$ frequency bins adjacent to $8 \mathrm{~Hz}$. The 10 epochs with the highest estimated noise were then discarded. The remaining 240 epochs were concatenated and averaged in 16 -sec sweeps. This provided a frequency resolution of $1 / 16$ $\mathrm{Hz}$ for all further analyses.

Response amplitudes were calculated at 4 and $8 \mathrm{~Hz}$ for each subject and for the grand average response. Background noise was estimated using $161 / 16$ th $\mathrm{Hz}$ frequency bins above and below each frequency. Because background noise is also present in response frequency bins, in cases where the signalto-noise ratio is low, response amplitude can be significantly overestimated. This overestimation can be modeled with a double-exponential function (Picton et al. 2005):

$$
1+0.965 \mathrm{e}^{-1.34 \mathrm{x}}+0.078 \mathrm{e}^{-0.285 \mathrm{x}}
$$

where $x$ is equal to the estimated signal-to-noise ratio of the measurement. Response amplitudes were scaled by the reciprocal of this factor, which ranged from 0.83 to 0.97 across blocks.

The significance of the grand average response at each level of asymmetry was analyzed using an $\mathrm{F}$ test to compare the power of the integrated components $(4$ and $8 \mathrm{~Hz}$ ) with the power of the underlying electrophysiologic noise across the $161 / 16$ th $\mathrm{Hz}$ frequency bins above and below each frequency. Next, the effects of asymmetry level and reference level on the amplitudes of (individual subject) responses at 4 and $8 \mathrm{~Hz}$ were assessed using both one- and two-way repeated measures analyses of variance (ANOVAs). The one-way ANOVA was used to assess changes in response amplitude across the five asymmetry levels with an 80 $\mathrm{dB}$ reference. The two-way ANOVA was used to assess changes in response amplitude across three levels of asymmetry $(0,10$, and $20 \mathrm{~dB}$ ) and two reference levels ( 80 and $60 \mathrm{dBA})$. An alpha level of 0.05 was used in all analyses. Greenhouse-Geisser corrections were used where sphericity could not be assumed. When testing the significance of the 4 and $8 \mathrm{~Hz}$ responses (at each of the nine levels), a Bonferroni correction was used to control type I error $(0.05 / 18=0.0028)$. The data collected from one participant were discarded because noise from muscle activity contaminated the recordings, as were the data collected from another participant who failed to complete the experiment. What follows is based on the data obtained from the remaining 13 listeners.

\section{RESULTS}

Figure 1 shows a sample of steady state responses over $1 \mathrm{sec}$ for three conditions (80:80, 60:60, and 80:60) for a single participant. Shaded and unshaded regions of the plots indicate stimulus periods during which the IAC of the noise stimulus was +1 and 0 , respectively. For the $80 \mathrm{dBA}$ reference condition with a $0-\mathrm{dB}$ interaural level difference (Fig. 1, top), an $8 \mathrm{~Hz}$ periodicity is clearly evident. It is also noteworthy that ASSR waves after changes of IAC from +1 to 0 appeared to be larger than those to changes of IAC from 0 to +1 (Fig. 1; see also Fig. 6 in Dajani \& Picton [2006]). For the $60 \mathrm{dBA}$ reference condition with no interaural level difference, the $8 \mathrm{~Hz}$ response remains evident, again with some tendency for response amplitudes to be greater after the change from correlated to uncorrelated noise (Fig. 1, middle). Finally, for the $80 \mathrm{dBA}$ reference condition with a $20-\mathrm{dB}$ interaural level difference (Fig. 1, bottom), amplitudes remained larger after the change from correlated to uncorrelated noise, although the periodic response was smaller and more noisy.

Data for the 4 and $8 \mathrm{~Hz}$ response components were analyzed separately. The neural responses at 4 and $8 \mathrm{~Hz}$ proved to be very different from each other. Figure 2 shows grand average response amplitudes at $4 \mathrm{~Hz}$ as a function of asymmetry level (abscissa) and reference level (parameter). The grand average response was significant at $4 \mathrm{~Hz}$ for every stimulus condition with an $80 \mathrm{dBA}$ reference, except 80:50 (reference ear:variable ear; dBA), $F(2,32)=6.68, p=0.004$ (i.e., $p>0.0028$ ). There were no significant grand average responses at $4 \mathrm{~Hz}$ with a 60 or $40 \mathrm{dBA}$ reference (i.e., $60: 60, F[2,32]=4.26, p=0.02296$; $60: 50, F[2,32]=4.76, p=0.01555 ; 60: 40, F[2,32]=5.26$, $p=0.0106$; and 40:40, $F[2,32]=4.85, p=0.01447$ ); this can be attributed to increased phase variability in the 40 and $60 \mathrm{~dB}$ reference conditions (Fig. 2), which is evidenced by large amplitude reductions relative to the individual subject response amplitude averages (see Fig. 3).

Figure 3 shows average individual subject response amplitudes at $4 \mathrm{~Hz}$ as a function of asymmetry level and reference level. There was no main effect of asymmetry, as confirmed by a two-way $(2 \times 3)$ repeated-measures ANOVA of the effects of reference level ( 80 and $60 \mathrm{dBA}$ ) and asymmetry on the amplitude of the neural response at $4 \mathrm{~Hz}$ at three levels of asymmetry $(0,10$, and $20 \mathrm{~dB})$ across all participants, $F(2,24)=$ $0.997, p=0.384$. Inspection of Figure 3 also suggests that there was no main effect of reference level, and this was confirmed statistically, $F(1,12)=0.431, p=0.524$. There was also no interaction between asymmetry and reference level, $F(2,24)=0.473, p=0.629$.

Grand average responses at $8 \mathrm{~Hz}$ (not shown) were significant at every level of asymmetry except $40 \mathrm{~dB}, F(2,32)=$ 5.23, $p=0.011$. All grand average responses were significant with the 60 and $40 \mathrm{dBA}$ references, indicating that binaural 


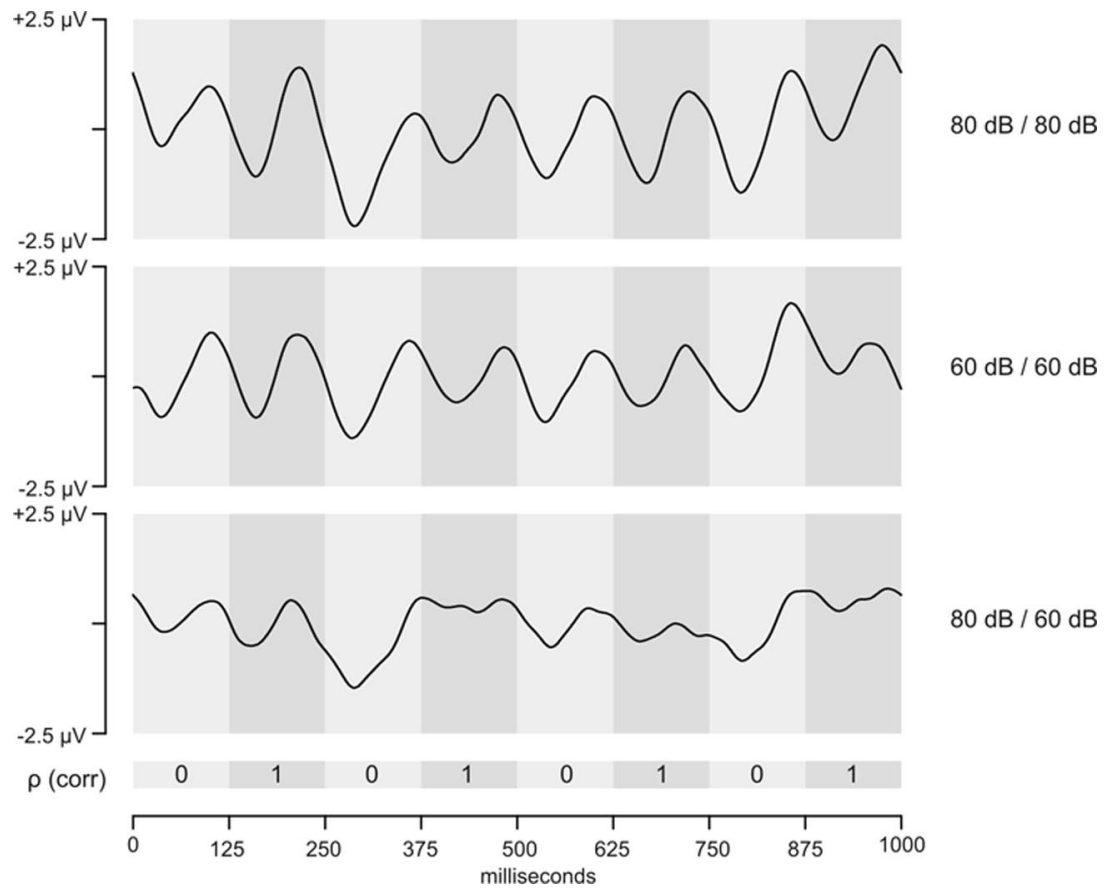

Fig. 1. Average responses from an individual subject, shown for three stimulus level conditions. Stimulus levels (reference ear/variable ear, $d B A$ ) are indicated on the right side. Unshaded areas represent periods during which the IAC was 0 , and shaded areas represent periods during which the IAC was +1 .

integration was still occurring for stimulus levels as low as 40:40 and 60:40.

Average individual subject response amplitudes at $8 \mathrm{~Hz}$ are shown in Figure 4, using the same conventions as those in Figure 3. Figure 4 shows that for the $8 \mathrm{~Hz}$ response, unlike the $4 \mathrm{~Hz}$ response, there was a significant main effect of asymmetry, as indicated by the inverse relationship between response amplitude and magnitude of the asymmetry. This was confirmed by a two-way $(2 \times 3)$ repeated-measures ANOVA examining the effects of reference level ( 80 and $60 \mathrm{dBA})$ and asymmetry on the amplitude of the neural response at $8 \mathrm{~Hz}$ at three levels of asymmetry $(0,10$, and $20 \mathrm{~dB})$ across all participants, $F(2,24)=58.258, p<0.0001$. A post hoc $t$ test

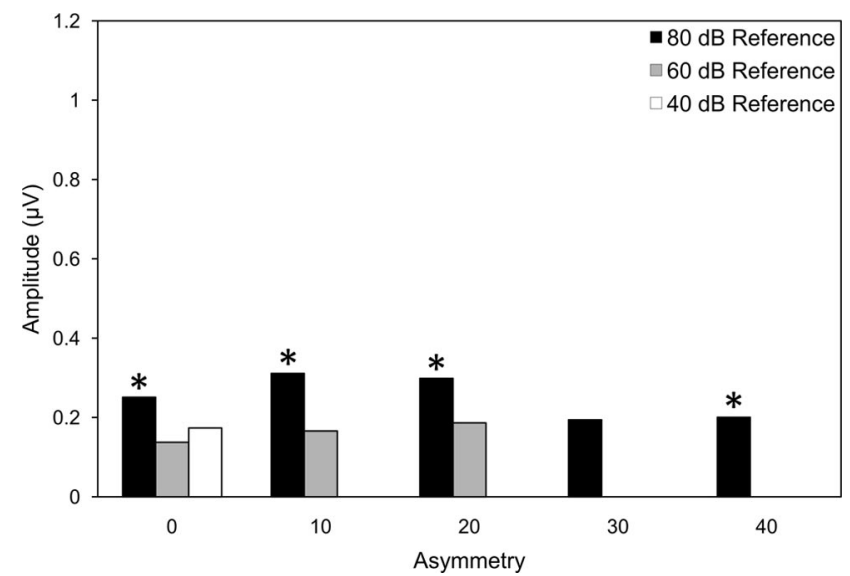

Fig. 2. Amplitudes of grand average responses at $4 \mathrm{~Hz}$ for all stimulus conditions plotted as a function of interaural level difference (abscissa). Stimulus reference level $(80,60$, and $40 \mathrm{dBA})$ is indicated by shading as shown in the legend at upper right. Asterisks denote that the grand average response was significantly greater than the noise floor of the recording. revealed that there was a significant drop in amplitude with even a $10 \mathrm{~dB}$ asymmetry $(80: 70), t(12)=6.4007, p<0.0001$.

There was also a significant effect of reference level, $F(1,12)=6.122, p=0.029$, which is shown by the vertical offset of the two data series in Figure 4. There was no significant interaction between reference level and asymmetry, $F(2,24)=1.567, p=0.229$. In Figure 4, this is expressed as the 80 and $60 \mathrm{dBA}$ data series having parallel trends across asymmetry level.

A second two-way $(2 \times 2)$ repeated-measures ANOVA was used to examine the effects of level and asymmetry for the two lower levels (60 and $40 \mathrm{dBA})$ with two levels of asymmetry $(0$ and $20 \mathrm{~dB})$. As with the higher-level analysis, there was a

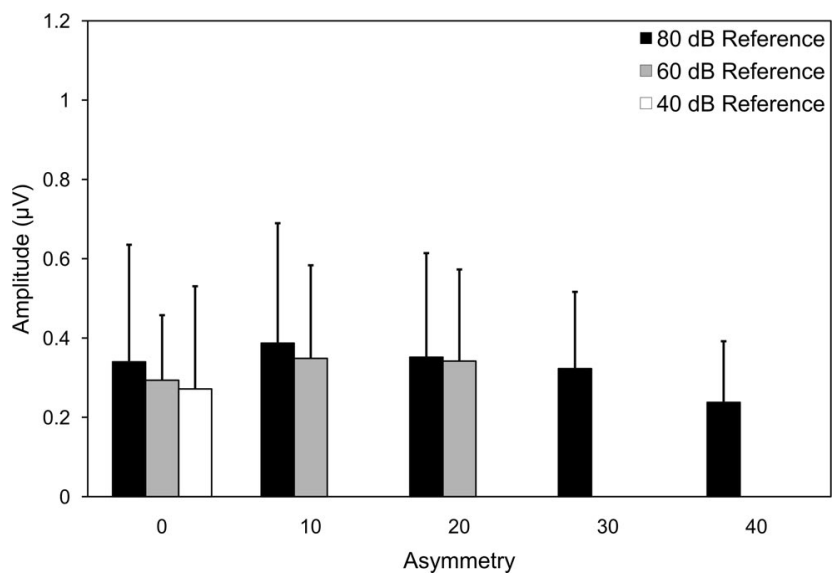

Fig. 3. Average of individual subject response amplitudes at $4 \mathrm{~Hz}$ for all stimulus conditions. Stimulus reference level $(80,60$, and $40 \mathrm{dBA})$ is indicated by shading as shown in the legend at upper right. Error bars are SDs. Note that the mean response amplitudes are independent of the asymmetry of stimulus levels at the two ears (interaural level difference, in $\mathrm{dB}$ ). 


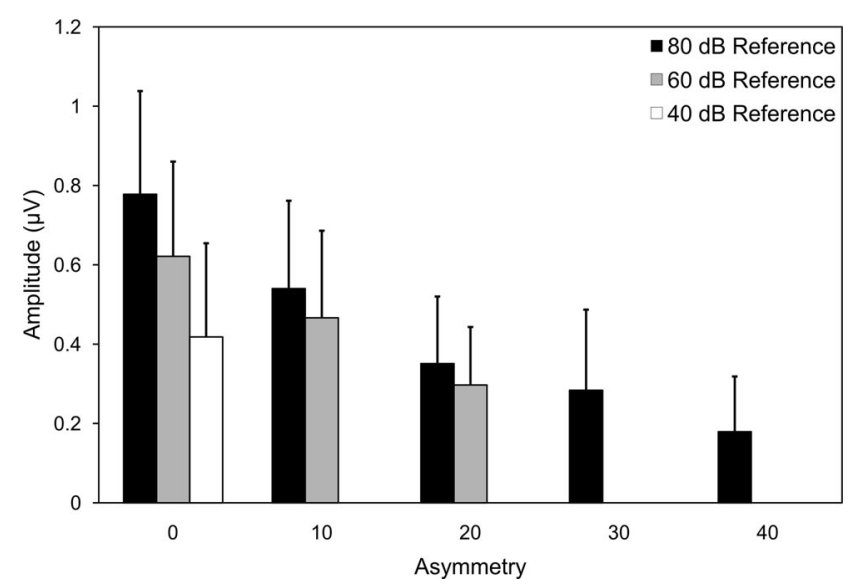

Fig. 4. Average of individual subject response amplitudes at $8 \mathrm{~Hz}$ for all stimulus conditions. Other details as for Figure 3. Note that response amplitude declines for the $80 \mathrm{~dB}$ reference level condition as a function of interaural level difference and does so over a $40 \mathrm{~dB}$ range. The $60 \mathrm{dBA}$ reference data follow the same trend over the $20 \mathrm{~dB}$ asymmetry levels tested.

significant effect of asymmetry, $F(1,12)=27.066, p<0.0001$, and level, $F(1,12)=8.753, p=0.012$, but no significant interaction, $F(1,12)=1.184, p=0.298$.

The effect of asymmetry was investigated further by a one-way repeated-measures ANOVA on the $8 \mathrm{~Hz}$ response amplitudes with a reference level of $80 \mathrm{~dB}$. In this analysis, five levels of asymmetry were assessed: $0,10,20,30$, and $40 \mathrm{~dB}$. Again, a significant main effect of asymmetry was revealed, $F(4,48)=51.979, p<0.0001$. A test of within-subjects contrasts indicated strong linear, $F(1,12)=90.657, p<$ 0.0001 , and quadratic trends, $F(1,12)=15.654, p=0.002$. The following quadratic trendline $\left(R^{2}=0.9933\right)$ indicated that response amplitude was reduced by $50 \%$ with an asymmetry of $18 \mathrm{~dB}$.

$$
y=0.0003 x^{2}-0.0256 x+0.7726
$$

\section{DISCUSSION}

The purpose of this study was to examine the effect of interaural level differences on the ASSR evoked in normal listeners by periodic changes in the IAC of a noise stimulus. Using the general methods of Dajani and Picton (2006) who first developed this paradigm, we confirmed the existence of significant 4 and $8 \mathrm{~Hz}$ response components driven by a $4 \mathrm{~Hz}$ cycle of IAC $=0 /+1$ change. The absolute values of the 4 and $8 \mathrm{~Hz}$ responses seen with $0 \mathrm{~dB}$ asymmetry in level at the ears ( $\approx 0.35$ and $0.8 \mu \mathrm{V}$, respectively, Figs. 2 and 4 ) are quite close to those previously reported by Dajani and Picton for the same stimulus conditions $(\approx 0.5$ and $0.85 \mu \mathrm{V}$, respectively, their Figs. 3 and 5).

This study had three main findings. The first was that the 8 $\mathrm{Hz}$ response component had a strong and orderly sensitivity to interaural level differences, for both 60 and $80 \mathrm{dBA}$ reference conditions (Fig. 4), whereas the $4 \mathrm{~Hz}$ response component did not (Fig. 3). Dajani and Picton (2006) similarly found a linear sensitivity to correlation strength in the $8 \mathrm{~Hz}$ response but not the $4 \mathrm{~Hz}$ response (their Fig. 8). The reasons for this difference are unclear. Our working hypothesis is that the $8 \mathrm{~Hz}$ response is driven by the rate of change in IAC, which in the $4 \mathrm{~Hz}$ paradigm occurs eight times per second. The origin of the $4 \mathrm{~Hz}$ response is unknown but may reflect differential responses to changes of IAC from +1 to 0 and from 0 to +1 (Chait et al. 2005); the oscillation occurs at $4 \mathrm{~Hz}$.

What is problematic for this view is that the $8 \mathrm{~Hz}$ response shows a strong sensitivity to interaural level asymmetry and degree of interaural correlation, whereas the $4 \mathrm{~Hz}$ response does not. If the two measures are different components of the same (interaural phase) coincidence detection mechanism, then one might have expected them to be equivalently sensitive to interaural level asymmetry or interaural correlation. However, it is important to make the distinction between phase-sensitive brain stem neurons involved in coincidence detection and the cortical processes underlying the evoked responses. The lack of sensitivity at $4 \mathrm{~Hz}$ does not imply a lack of sensitivity at the level of the brain stem, particularly because interaural asymmetry sensitivity is evident at $8 \mathrm{~Hz}$. A more likely explanation is that the energy at $4 \mathrm{~Hz}$ reflects the difference between cortical responses to changes of IAC from +1 to 0 and from 0 to +1 , and this difference is relatively constant across the levels of interaural level asymmetry used in this study.

Behavioral experiments reveal greater perceptual sensitivity to decreases in interaural correlation (e.g., changes in IAC from +1 to 0 ) than to increases (Boehnke et al. 2002; Chait et al. 2005). The present ASSR data suggest an electrophysiological correlate of this behavior in that ASSR waves after changes of IAC from +1 to 0 tend to be larger than those to changes of IAC from 0 to +1 (Fig. 1; see also Figure 6 in Dajani \& Picton [2006]), especially when the presentation levels are asymmetric (Fig. 1, bottom panel). However, it would be simplistic to assume that each ASSR deflection is the direct consequence of the immediately preceding correlation change. Dajani and Picton's (2006) 1 and $2 \mathrm{~Hz}$ data clearly show a triphasic $\mathrm{P} 1-\mathrm{N} 1-\mathrm{P} 2$ response to each stimulus change, with the N1 occurring at a latency of 122 msecs. If a similar triphasic response underlies the $8 \mathrm{~Hz}$ activity in this study, the larger deflections giving rise to the energy at $4 \mathrm{~Hz}$ could be enhanced $\mathrm{N} 1-\mathrm{P} 2$ responses to the earlier 0 to +1 IAC change. This interpretation is problematic in several respects: the average latencies of the negative and positive deflections are 162 and 225 msecs, respectively (40 msecs later than the average N1 and P2 latencies at $1 \mathrm{~Hz}$ in Dajani \& Picton [2006]); a larger $\mathrm{N} 1-\mathrm{P} 2$ to the 0 to +1 change would be odd given that behavioral sensitivity is generally lower to this change; and studies using presentation rates slow enough to clearly distinguish individual components have found the N1-P2 to be largest in response to +1 to 0 IAC changes (Chait et al. 2005). Similar results have also been obtained for other diotic to dichotic changes (see Fig. 11 in Ross [2008]). Nevertheless, deflections in the ASSR may be fully or partially evoked by correlation changes preceding the most immediate, so it is not safe to assume that the larger deflections giving rise to the energy at $4 \mathrm{~Hz}$ reflect better sensitivity to decreases in correlation.

The second major finding of the study was that the $8 \mathrm{~Hz}$ response was far more sensitive to changes in interaural asymmetry than changes in absolute level. Responses to low-level symmetric conditions were larger than responses to high-level asymmetric conditions (e.g., 40:40 > 80:60 and 60:60 > 80:70; see Fig. 4). Even an asymmetry as small as 
$10 \mathrm{~dB}$ gave rise to a significant decrease in $8 \mathrm{~Hz}$ response amplitude. This bodes well for future applications of the 8 $\mathrm{Hz}$ response to study the effects of hearing asymmetries on binaural temporal integration; the response is very sensitive to asymmetry and can be recorded over a range of levels.

The third major finding of this study was the form taken by the $8 \mathrm{~Hz}$ response's sensitivity to interaural level differences (Fig. 4). For both reference levels ( 80 and $60 \mathrm{dBA}$ ), the $8 \mathrm{~Hz}$ response amplitude falls quickly over the first $20 \mathrm{~dB}$ of interaural level difference. For the $80 \mathrm{dBA}$ reference condition (the only one tested with a wider range of asymmetries), the 8 $\mathrm{Hz}$ response then falls more slowly with further increments in the asymmetry and becomes statistically indistinguishable from background for an asymmetry of $40 \mathrm{~dB}$. A post hoc quadratic fit of the $80 \mathrm{dBA}$ reference data (accounting for $99 \%$ of the data variance) showed the response to be already at half strength for an asymmetry of only $18 \mathrm{~dB}$, even though the binaural integration mechanism can tolerate an interaural level asymmetry of at least $30 \mathrm{~dB}$. Interestingly, Blodgett et al. (1962) found that a binaural masking level difference (monaural signal with in-phase noise, relative to monaural signal with monaural noise) was present for presentation level asymmetries up to $30 \mathrm{~dB}$ and was reduced by half with an asymmetry of 18 $\mathrm{dB}$, suggesting that the interaural correlation ASSR and the masking level difference may depend on a common neural substrate. The $18 \mathrm{~dB}$ midpoint value is also similar to the clinical "Belfast Rule of Thumb," which recommends that in cases of asymmetric hearing loss, restoration should target better than $15 \mathrm{~dB}$ interaural asymmetry to optimize behaviorally measured binaural integration (Smyth \& Patterson 1985; Nia \& Bance 2001). However, Wilson et al. (1985) recorded speech masking level differences with a $40 \mathrm{~dB}$ interaural presentation level asymmetry (speech out-of-phase versus inphase, with masking noise always in-phase) and found that masking level differences were only $26 \%$ smaller on average with an $18 \mathrm{~dB}$ asymmetry for young normal-hearing subjects. Perhaps, electrophysiologic responses to interaural correlation changes from 0 to +1 and +1 to 0 are linearly related to some masking level differences (e.g., monaural tone thresholds in binaural in-phase masking noise versus monaural masking noise) and not others (masked binaural speech reception thresholds with in-phase versus out-of-phase speech). Nonetheless, the functional implications of the $18 \mathrm{~dB}$ midpoint in the amplitude of the $8 \mathrm{~Hz}$ ASSR should be interpreted with caution.

Ultimately, the importance of optimal binaural temporal integration should be considered in light of the value of binaural processing for issues of practical significance, such as hearing speech in noisy environments. For instance, a surgical intervention that improves the hearing in one ear at the expense of hearing symmetry may reduce access to binaural temporal cues but may provide access to more speech information and thus be warranted. However, binaural temporal integration is an important auditory function (Boothroyd 2006) and is related to outcomes (Gatehouse \& Akeroyd 2006). Also, small asymmetries $(\sim 10 \mathrm{~dB})$ can significantly reduce the amplitude of the binaural correlation ASSR (as shown in this study) and reduce masking level differences for tones (Blodgett et al. 1962) and speech (Wilson et al. 1985); these asymmetries could probably be eliminated in many cases without reducing access to speech information.
In this study, interaural correlation ASSRs were recorded at many levels of presentation asymmetry and multiple reference levels. This procedure could be refined for clinical assessment of individuals with asymmetric loss. For example, ASSRs could be recorded with a fixed level in the poorer ear and a small set of levels in the better ear. Also, instead of recording separate responses at each level of asymmetry, the intensity of the noise stimulus in the better ear could be continuously ramped. The amplitude of the binaural ASSR could then be plotted as a function of interaural presentation level symmetry, with the peak of the function showing the optimal interaural balance (which might change as a function of time after a treatment that reduces or increases asymmetries in hearing). These approaches will be explored in future studies.

In summary, our data show that an $8 \mathrm{~Hz}$ response to $4 \mathrm{~Hz}$ cycles of interaural correlation change, but not a concurrent 4 $\mathrm{Hz}$ response, is sensitive to interaural level differences. The amplitude of the $8 \mathrm{~Hz}$ response becomes statistically insignificant for interaural level differences $>30 \mathrm{~dB}$. This binaurally sensitive ASSR may be ideally suited for assessing the effects of hearing asymmetries on binaural temporal integration.

\section{ACKNOWLEDGMENTS}

The authors thank Dr. Hilmi Dajani for his generous advice and help with stimulus design.

This work was supported by The Hearing Foundation of Canada, the Capital Health District Authority Research Fund, the Canada Research Chairs program (to A. J. N.), and NSERC (to A. J. N., D. P., and M. B.).

An earlier version of this article was submitted in partial fulfillment of the first author's BSc (Hons.) degree in Neuroscience at Dalhousie University.

Address for all correspondence: Steve J. Aiken, School of Human Communication Disorders, Dalhousie University, 5599 Fenwick Street, PR Level, Halifax, Nova Scotia, Canada B3H 1R2. E-mail: steve.aiken@dal.ca.

Received September 4, 2009; accepted June 3, 2010.

\section{REFERENCES}

Bernstein, L. R., \& Trahiotis, C. (1992). Discrimination of interaural envelope correlation and its relation to binaural unmasking in the high frequencies. J Acoust Soc Am, 91, 306-316.

Blauert, J., \& Lindemann, W. (1986). Spatial mapping of intracranial auditory events for various degrees of interaural coherence. J Acoust Soc Am, 79, 806-813.

Blodgett, H. C., Jeffress, L. A., Whitworth, R. H. (1962). Effect of noise at one ear on the masked threshold for tone at the other. J Acoust Soc Am, 34, 979-981.

Bocca, E., \& Antonelli, A. R. (1976). Masking level difference: Another tool for the evaluation of peripheral and cortical defects. Audiology, 15, 480-487.

Boehnke, S. E., Hall, S. E., Marquardt, T. (2002). Detection of static and dynamic changes in interaural correlation. J Acoust Soc Am, 112, 1617-1626.

Boothroyd, A. (2006). Characteristics of listening environments: Benefits of binaural hearing and implications for bilateral management. Int $J$ Audiol, 45, S12-S19.

Bronkhorst, A. W., \& Plomp, R. (1989). Binaural speech intelligibility in noise for hearing-impaired listeners. J Acoust Soc Am, 86, 1374-1383.

Browning, G. G. (1993). Reporting the benefits from middle ear surgery using the Glasgow Benefit Plot. Am J Otol, 14, 135-140.

Browning, G. G., Gatehouse, S., Swan, I. R. (1991). The Glasgow Benefit Plot: A new method for reporting benefits from middle ear surgery. Laryngoscope, 101, 180-185.

Chait, M., Poeppel, D., de Cheveigné, A., et al. (2005). Human auditory cortical processing of changes in interaural correlation. J Neurosci, 25, $8518-8527$. 
Culling, J. F., Colburn, H. S., Spurchise, M. (2001). Interaural correlation sensitivity. J Acoust Soc Am, 110, 1020-1029.

Dajani, H. R., \& Picton, T. W. (2006). Human auditory steady-state responses to changes in interaural correlation. Hear Res, 219, 85-100.

Durlach, N. I., Braida, L. D., Ito, Y. (1986). Towards a model for discrimination of broadband signals. $J$ Acoust Soc Am, 80, 63-73.

Durlach, N. I., Gabriel, K. J., Colburn, H. S., et al. (1986). Interaural correlation discrimination. II. Relation to binaural unmasking. $J$ Acoust Soc Am, 79, 1548-1557.

Durlach, N. I., Thompson, C. L., Colburn, H. S. (1981). Binaural interaction of impaired listeners. A review of past research. Audiology, $20,181-211$.

Gatehouse, S., \& Akeroyd, M. (2006). Two-eared listening in dynamic situations. Int J Audiol, 45, S120-S124.

Gatehouse, S., \& Noble, W. (2004). The speech, spatial and qualities of hearing scale. Int $J$ Audiol, 43, 85-99.

Halliday, R., \& Callaway, E. (1978). Time shift evoked potentials (TSEPs): Method and basic results. Electroenceph Clin Neurophysiol, 45, 118-121.

Hari, R., Aittoniemi, K., Järvinen, M. L., et al. (1980). Auditory evoked transient and sustained magnetic fields of the human brain localization of neural generators. Exp Brain Res, 40, 237-240.

Häusler, R., Colburn, S., Marr, E. (1983). Sound localization in subjects with impaired hearing. Spatial discrimination and interaural-discrimination tests. Acta Otolaryngol Suppl, 400, 1-62.

Jain, M., Gallagher, D. T., Koehnke, J., et al. (1991). Fringed correlation discrimination and binaural detection. J Acoust Soc Am, 90, 1918-1926.

Koehnke, J., Colburn, H. S., Durlach, N. I. (1986). Performance in several binaural-interaction experiments. J Acoust Soc Am, 79, 1558-1562.

Koehnke, J., Culotta, C. P., Hawley, M. L., et al. S. (1995). Effects of reference interaural time and intensity differences on binaural performance in listeners with normal and impaired hearing. Ear Hear, 16, 331-353.

Krumbholz, K., Magezi, D. A., Moore, R. C. (2009). Binaural sluggishness precludes temporal pitch processing based on envelope cues in conditions of binaural unmasking. J Acoust Soc Am, 125, 1067-1074.

McEvoy, L. K., Picton, T. W., Champagne, S. C., et al. (1990). Human evoked potentials to shifts in the lateralization of a noise. Audiology, 29, 163-180.

Moore, D. R. (1991). Anatomy and physiology of binaural hearing. Audiology, 30, 125-134.
Näätänen, R., \& Picton, T. (1987). The N1 wave of the human electric and magnetic response to sound: A review and an analysis of the component structure. Psychophysiol, 24, 375-425.

Nia, J., \& Bance, M. (2001). Effects of varying unilateral conductive hearing losses on speech-in-noise discrimination: An experimental study with implications for surgical correction. Otol Neurotol, 22, 737-744.

Olsen, W. O., Noffsinger, D., Carhart, R. (1976). Masking level differences encountered in clinical populations. Audiology, 15, 287-301.

Phillips, D. P. (2008). A perceptual architecture for sound lateralization in man. Hear Res, 238, 124-132.

Picton, T. W., Dimitrijevic, A., Perez-Abalo, M., et al. (2005). Estimating audiometric thresholds using auditory steady-state responses. $J$ Am Acad Audiol, 16, 140-156.

Picton, T. W., McEvoy, L. K., Champagne, S. C. (1991). Human evoked potentials and the lateralization of sound. Acta Otolaryngol Suppl, 491, $139-144$.

Quaranta, A., \& Cervellera, G. (1974). Masking level difference in normal and pathological ears. Audiology, 13, 428-431.

Ross, B. (2008). A novel type of auditory responses: Temporal dynamics of $40-\mathrm{Hz}$ steady-state responses induced by changes in sound localization. J Neurophysiol, 100, 1265-1277.

Scherg, M., Vajsar, J., Picton, T. W. (1989). A source analysis of the late human auditory evoked potentials. J Cog Neurosci, 1, 336-355.

Smyth, G. D., \& Patterson, C. C. (1985). Results of middle ear reconstruction: Do patients and surgeons agree? Am J Otol, 6, 276-279.

Toner, J. G., \& Smyth, G. D. (1993). Comparison of methods of evaluating hearing benefit of middle ear surgery. J Laryngol Otol, 107, 4-5.

Wilson, J. R., \& Krishnan, A. (2005). Human frequency-following responses to binaural masking level difference stimuli. J Am Acad Audiol, $16,184-195$.

Wilson, M. J., Kelly-Ballweber, D., Dobie, R. A. (1985). Binaural interaction in auditory brainstem responses: Parametric studies. Ear Hear, 6, 80-88.

Wong, W. Y. S., \& Stapells, D. R. (2004). Brain stem and cortical mechanisms underlying binaural masking level differences in humans: An auditory steady-state response study. Ear Hear, 25, 57-67.

Yin, T. C. T., Chan, J. C. K., Irvine, D. R. F. (1986). Effects of interaural time delays of noise stimuli on low-frequency cells in the cat's inferior colliculus. I. Responses to wideband noise. J Neurophysiol, 55, 280-300. 